Special issue of the 3rd International Conference on Computational and Experimental Science and Engineering (ICCESEN 2016)

\title{
An Artificial Neural Network-Based Estimation of Bremsstarahlung Photon Flux Calculated by MCNPX
}

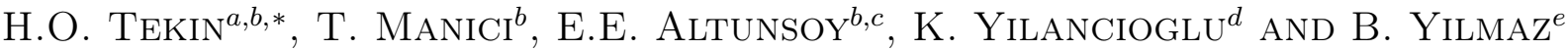

${ }^{a}$ Uskudar University, Vocational School of Health Services, Radiotherapy Department, İstanbul, Turkey

${ }^{b}$ Usküdar University, Medical Radiation Research Center (USMERA), İstanbul, Turkey

${ }^{c}$ Uskudar University, Vocational School of Health Service, Medical Imaging Department, Istanbul, Turkey

${ }^{d}$ Uskudar University, Faculty of Engineering and Natural Sciences,

Molecular Biology and Genetics Department, İstanbul, Turkey

${ }^{e}$ Okan University, Faculity of Medicine, Department of Radiology, Istanbul, Turkey

Bremsstrahlung has an important place in the field of experimental physics, especially for description of photon-matter interaction and for characterization and analysis of materials. Bremsstrahlung photon is created by a high-energy electron, deflected in the electric field of atomic nucleus. Bremsstrahlung is also important for experimental studies, not only in the field of nuclear physics and particle physics but also in the fields of solid state physics, applied physics and astrophysics. In recent years, Monte Carlo simulation has become a widely used method for calculations related to bremsstrahlung. On the other hand, predictions by using artificial neural network can be performed with high accuracy. This study aims at observing variation in the photon flux as unction of target thickness and at processing output data by using an artificial neural network. We achieved a high degree of compatibility between two different methods. This study suggests that artificial neural network is a powerful tool for prediction of Bremsstrahlung and for other scientific problems.
\end{abstract}

DOI: 10.12693/APhysPolA.132.967

PACS/topics: 07.05.Mh, 23.20.Nx, 87.10.R

\section{Introduction}

$\mathrm{X}$-rays are mainly produced by high energy electrons, which can be accelerated up to certain energy levels. When the electrons are bombarding a target material with high atomic number, x-rays can be generated. When the incoming electron penetrates into the target material, some electrons can pass through the nucleus due to its positive electric charge. In this process a part or all of their kinetic energy can be lost and the X-rays, generated from this lost energy, are emitted. These Xrays are called bremsstrahlung photons. The energy of the bremsstrahlung photons can be in the range from zero to the energy of incoming electron. The schematic view of bremsstrahlung process is shown in Fig. 1.

The generated bremsstrahlung photon flux strongly depends on incoming electron beam energy, type of target material and target thickness. Nowadays, Monte Carlo simulations are widely used in various fields, such as nuclear physics, medical physics etc. This method can be also applied to estimation of the bremsstrahlung photon flux.

For such applications, several models have been developed, such as MCNP, GEANT4, EGNSnrc, Penelope and FLUKA. This study uses a prediction method based on artificial neural network (ANN). Various ANN prediction and estimation studies can be found in literature [1-5].

\footnotetext{
* corresponding author; e-mail: huseyinozan. tekin@uskudar.edu.tr
}

Previous studies have shown the applicability of ANN to prediction of bremsstrahlung photon yield from aluminum target of different thicknesses. According to the study, the photon flux from $\mathrm{Al}$ target of varied thickness has been estimated successfully using the developed ANN model. Thus bremsstrahlung photon beam flux, which is an important parameter in photonuclear reaction experiments, could be estimated [6-10].

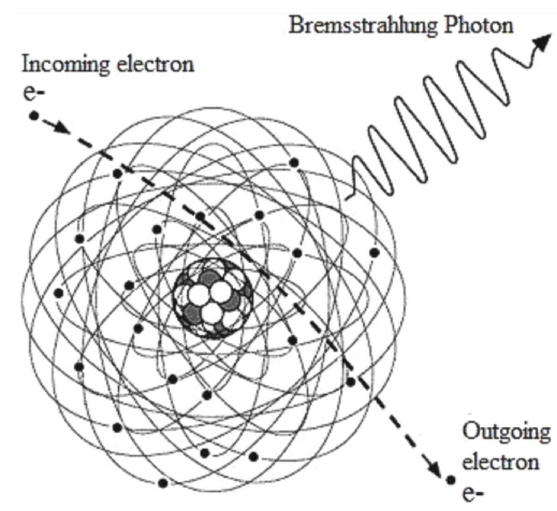

Fig. 1. The schematic view of bremsstrahlung process.

\section{Materials and methods}

Artificial neural network is an information process that is inspired by human brain and nervous system, which use a complex brain information network [11]. The ANN can supply a reliable information. ANN includes an input layer, one or more hidden layers and an output layer. The structure of ANN is shown in Fig. 2. The input and 
output data are considered as information processing systems that have the abilities to learn, recall and generalize from sample training data. The number of neurons in a layer and the number of layers depend on the the complexity of the studied system.

The neurons in the input layer take the data and transfer it to neurons in the first hidden layer through the weighted links. In the hidden layers the data are mathematically processed and the result is transferred to the neurons of the next layer. The last layer provides the output of the network. In a hidden layer, the $j$ th neuron processes the incoming data $x_{i}$ by: (i) calculating the weighted sum and adding a "bias" term $\theta_{j}$, according to Eq. (1)

$$
\text { net } j=\sum_{i=1}^{w} x_{i} w_{i j}+\theta_{j}, j=1,2, \ldots, n,
$$

(ii) transforming the net $j$ through a suitable mathematical "transfer function", and (iii) transferring the result to neurons in the next layer. Various transfer functions are available, however the most commonly used is the sigmoid function [12]:

$$
f(x)=\frac{1}{1+\mathrm{e}^{-x}} .
$$

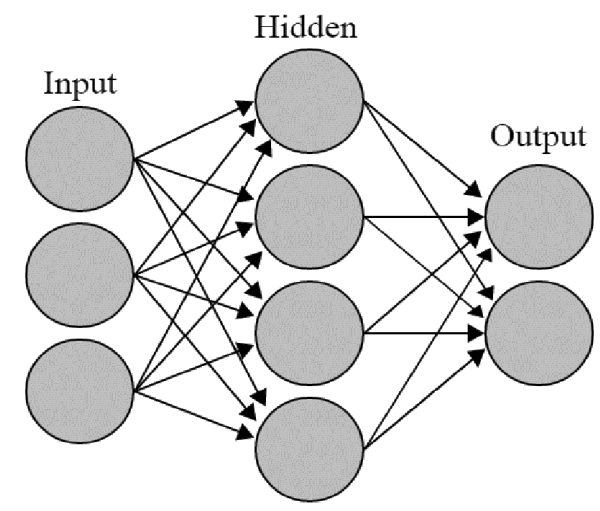

Fig. 2. The structure of an artificial neural network.

In this study, MCNPX (version 2.6.0) has been used as the Monte Carlo code. MCNPX is a general purpose radiation transport code for modeling the interaction of radiation with materials, which also tracks all particles at all energies. MCNPX is fully three-dimensional and it utilizes extended nuclear cross section libraries and uses physics models for particle types [13]. MCNPX is a powerful tool for various kinds of applications. The capabilities of MCNPX have been investigated in literature. Various applications using MCNPX code have been found in literature [14-19].

Electron-ray source at $10 \mathrm{MeV}$ was defined in data card section of MCNPX input, considering different variables, such as CEL, ERG, DIR, POS, and PAR. The geometric center of the detection cell has been considered for location of point source. Each variable has different abilities during Monte Carlo simulation. In the present study, variables commanded source cell, energy, direction, source position and particle type, respectively. Thus, we obtained the bremsstrahlung photon flux from the MCNPX Monte Carlo code. The obtained results have been considered as input parameters of the ANN model. Target thickness and electron beam energy have been selected as the input parameters in ANN model. The RapidMiner software was used for predictive ANN modeling [20].

\section{Results and discussion}

In this study, we calculated the bremsstrahlung photon flux for $2 \mu \mathrm{m}$ tantalum (Ta) target by using MCNPX code. The results are shown in Fig. 3. We have considered tantalum as the target material with thicknesses of $2 \mu \mathrm{m}, 4 \mu \mathrm{m}, 6 \mu \mathrm{m}, 8 \mu \mathrm{m}$ and $10 \mu \mathrm{m}$. $10 \mathrm{MeV}$ mono energetic electron beam was directed onto Ta target material. We have used $80 \%$ of photon flux data, obtained from Monte Carlo simulation as the training data for ANN.

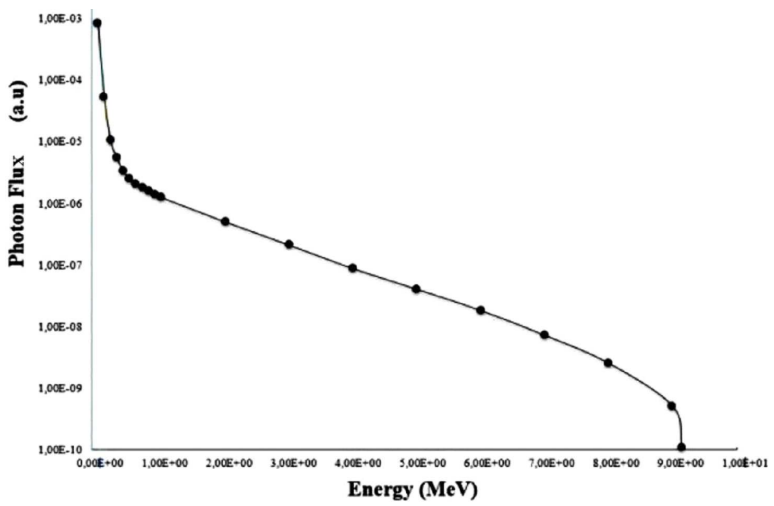

Fig. 3. Bremsstrahlung photon flux, obtained using MCNPX

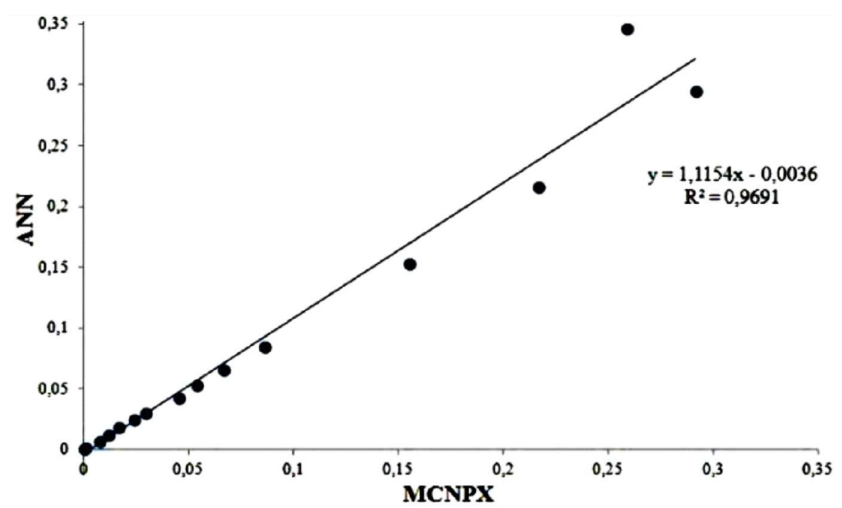

Fig. 4. Correlation between photon fluxes obtained using ANN and MCNPX.

Afterwards, we have used the rest $20 \%$ of data, obtained from the same Monte Carlo simulation, as the test data for evaluation of the predictions. The photon flux 
from Ta target for a certain thickness has been estimated by using ANN model. It is demonstrated in Fig. 4 that a good correlation with an $R^{2}=0.9691$ has been obtained between photon flux, calculated by MCNPX, and photon flux estimated by ANN. According to previous studies, found in literature, similar results have been already shown. Investigation of correlation of EGSnrc and ANN model has been studied by Akkurt et al. [10] and a good correlation between models has been also shown.

It is suggested that MCNPX is a powerful and effective tool for investigation of bremsstrahlung photon flux, where experimental results are not available. On the other hand, it is demonstrated in this study that ANN is a powerful prediction model that can be used for different future investigations in similar fields.

\section{References}

[1] Y. Ozcanli, F. Kosovali Cavus, M. Beken, Acta Phys. Pol. A 130, 444 (2016)

[2] M. Davraz, S. Kilincarslan, H. Ceylan, Acta Phys. Pol. A 128, B-184 (2015).

[3] N. Zeng, Z. Wang, H. Zhang, F.E. Alsaadi, Cogn. Comput. 8, 143 (2016).

[4] J. Liang, S.Y. Yuen, Cogn. Comput. 8, 693 (2016).

[5] Z. Tang, J. Lu, P. Wang, Cogn. Comput. 7, 731 (2015).

[6] I. Akkurt, K. Günoglu, H.O. Tekin, Z.N. Demirci, G. Yegin, N. Demir, Iranian J. Rad. Res. 10, 63 (2011).

[7] U. Kara, A. Mesbahi, I. Akkurt, Acta Phys. Pol. A 128, B-378 (2015).

[8] N. Demir, Z.N. Kuluozturk, I. Akkurt, Acta Phys. Pol. A 128, B-443 (2015).
[9] K. Gunoglu, N. Demir, I. Akkurt, Z.N. Demirci, Neural Comput. Applicat. 23, 1591 (2013).

[10] N. Demir, Z.N. Demirci, I. Akkurt, Radiat. Eff. Defects Solids 168, 372 (2013).

[11] J. Schmidhuber, Neural Networks 61, 85 (2015).

[12] F. Amato, A. López, E. María, P. Méndez, P. Vaňhara, A. Hampl, J. Havel, J. Appl. Biomed. 11, 47 (2013).

[13] RSICC Computer Code Collection, MCNPX User's Manual Version 2.4.0. Monte Carlo N-Particle Transport Code System for Multiple and High Energy Applications, 2002.

[14] H.O. Tekin, Sci. Technol. Nucl. Installations 2016 , 6547318 (2016).

[15] I. Akkurt, H.O. Tekin, A. Mesbahi, Acta Phys. Pol. A 128, B-332 (2015)

[16] H.O. Tekin, U. Kara, J. Communicat. Comput. 13 , 32 (2016)

[17] H.O. Tekin, T. Manici, C. Ekmekci, J. Health Sci. 4, 131 (2016).

[18] H.O. Tekin, V.P. Singh, T. Manici, Appl. Radiat. Isotop. 121, 122 (2017).

[19] I. Akkurt, J.-O. Adler, J.R.M. Annand, F. Fasolo, K. Hansen, L. Isaksson, M. Karlsson, P. Lilja, M. Lundin, B. Nilsson, C. Ongaro, A. Reiter, G. Rosner, A. Sandell, B. Schröder, A. Zanini, Phys. Med. Biol. 48, 3345 (2003).

[20] O. Ritthoff, R. Klinkenberg, S. Fisher, I. Mierswa, S. Felske, YALE: Yet another Learning Environment. LLWA'01 - Tagungsband der GI-WorkshopWoche Lernen-Lehren - Wissen Adaptivitat, Technical Report 763, University of Dortmund, Dortmund 2001, p. 84 . 